\title{
Physical parameters of a relativistic jet at very high redshift: the case of the blazar $\mathrm{J} 1430+4204$
}

\author{
P. Veres ${ }^{1,2}$, S. Frey ${ }^{3,5}$, Z. Paragi ${ }^{4,5}$, and L. I. Gurvits ${ }^{4}$ \\ 1 Dept. of Physics of Complex Systems, Eötvös University, Pázmány P. s. 1/A, 1117 Budapest, Hungary \\ e-mail: veresp@elte.hu \\ 2 Dept. of Physics, Bolyai Military University, PO Box 15, Budapest, 1581, Hungary \\ FÖMI Satellite Geodetic Observatory, PO Box 585, 1592 Budapest, Hungary \\ 4 Joint Institute for VLBI in Europe, PO Box 2, 7990 AA Dwingeloo, The Netherlands \\ 5 MTA Research Group for Physical Geodesy and Geodynamics, PO Box 91, 1521 Budapest, Hungary
}

Received 8 May 2010 / Accepted 23 June 2010

\section{ABSTRACT}

\begin{abstract}
Context. The high-redshift $(z=4.72)$ blazar J1430+4204 produced a major radio outburst in 2005. Such outbursts are usually associated with the emergence of a new component in the inner radio jet.

Aims. We searched for possible changes in the radio structure on milli-arcsecond angular scales, to determine physical parameters that characterise the relativistic jet ejected from the centre of this source.

Methods. We analysed 15-GHz radio interferometric images obtained with the Very Long Baseline Array (VLBA) before and after the peak of the outburst.

Results. We did not identify any significant new jet component over a period of 569 days. We estimated the Doppler factor, the Lorentz factor, and the apparent transverse speed of a putative jet component using three different methods. The likely small jet angle to the line of sight and our values of the apparent transverse speed are consistent with not detecting a new jet feature.
\end{abstract}

Key words. radio continuum: galaxies - galaxies: active - galaxies: jets - galaxies: individual: J1430+4204 techniques: interferometric

\section{Introduction}

Active galactic nuclei (AGNs) are thought to harbour supermassive (up to $\sim 10^{9.5} M_{\odot}$ ) black holes. Accretion onto these black holes is responsible for the extreme luminosity of AGNs over the whole electromagnetic spectrum. Part of the infalling matter may be transformed into jets ejected with relativistic speeds. The radio emission in radio-loud AGNs originates in these jets via synchrotron process. The appearance of the source strongly depends on the orientation of the jet axis with respect to viewing direction. If a jet points close to the line of sight toward the observer, its brightness is significantly enhanced. For a review of the unified schemes for radio-loud AGNs, see Urry \& Padovani (1995).

A particular class of AGNs are blazars. They show large and rapid brightness variations, from the radio to the gamma-ray regime. Among them, the BL Lac objects have no strong emission lines characteristic of other AGNs in the optical spectrum. The radio polarisation of the blazars could be high and variable. According to the models of AGNs (Urry \& Padovani 1995), we look at these objects from almost exactly the direction of their relativistic jets.

$\mathrm{J} 1430+4204(\mathrm{~B} 1428+4217)$ is a blazar with a flat radio spectrum, at an extremely high redshift, $z=4.72$ (Hook \& McMahon 1998). Long-term radio flux density monitoring at $15 \mathrm{GHz}$ revealed a significant brightening of J1430+4204, starting in 2004 and reaching its peak in 2005 (Worsley et al. 2006). The object increased its flux density by a factor of 3 in about 4 months (in the source rest frame). At the same time, a corresponding outburst was not detected in X-rays with the XMM-Newton satellite, although a long-term X-ray spectral variability is evident in the source (Worsley et al. 2006). The X-ray spectrum of J1430+4204 suggests a large amount of intrinsic absorption with neutral hydrogen column density $N_{\mathrm{H}} \simeq 10^{22}-10^{23} \mathrm{~cm}^{-2}$ (Worsley et al. 2004, 2006).

The radio structure of $\mathrm{J} 1430+4204$ at the milli-arcsecond (mas) scale is mainly compact as revealed by Very Long Baseline Interferometry (VLBI) imaging observations at $5 \mathrm{GHz}$ (Paragi et al. 1999; Helmboldt et al. 2007), and 2.3 and $8.4 \mathrm{GHz}$ (US Naval Observatory Radio Reference Frame Image Database, USNO RRFID ${ }^{1}$ ). A weak extension to the bright compact core is also seen in the W-SW direction. On scales three orders of magnitude larger, the central emission region is dominant as well, but there is also a jet component at a $\sim 4^{\prime \prime}$ angular distance from the brightness peak, in the W-NW direction (Fig. 2 of Cheung et al. 2008). The pc- and kpc-scale structures are therefore misaligned by $\sim 50^{\circ}$. Imaging with the Chandra satellite revealed X-ray emission also at the position of the kpc-scale radio jet component. This source has currently the highest redshift kpc-scale radio and X-ray jet known (Cheung et al. 2008).

Total flux density outbursts are usually followed by an emergence of a new jet component in the VLBI images of radio AGNs (e.g. Savolainen et al. 2002). By observing J1430+4204 after the brightening, we aimed at detecting a new component in a hope to have a zero-epoch point for a later measurement of its apparent proper motion. Due to the uniquely high redshift, the source

\footnotetext{
${ }^{1}$ http://rorf.usno.navy.mil/rrfid.shtml
} 


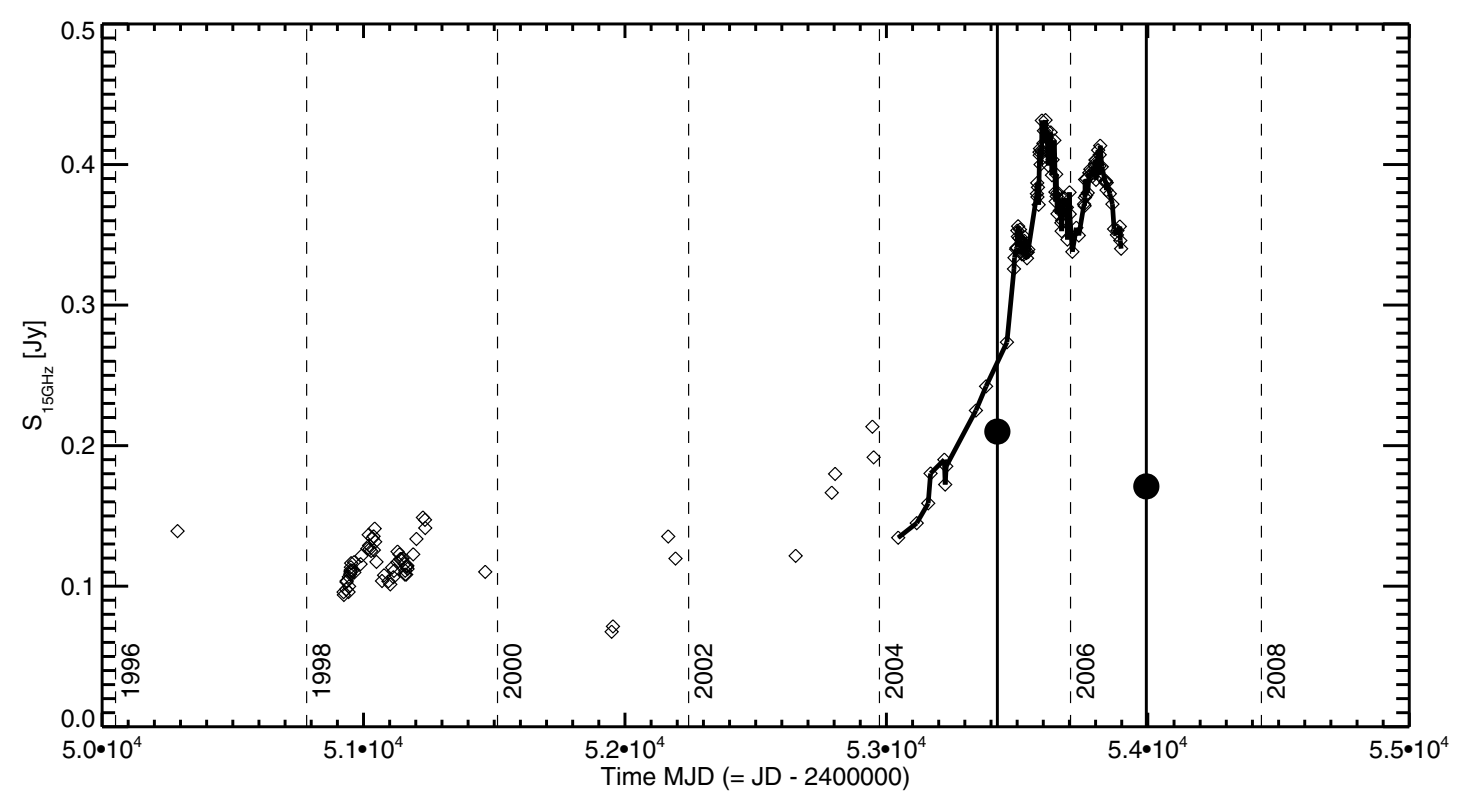

Fig. 1. The 15-GHz total flux density vs. time for J1430+4204 from the Ryle Telescope monitoring (G. Pooley, priv. comm.). The points are connected in the flaring phase for clarity. Solid vertical lines mark the times of the two VLBA observations. The filled circles correspond to the measured VLBI flux densities. Dashed lines mark calendar years as indicated. The typical flux density error bars are smaller or comparable in size with the symbols.

J1430+4204 would provide an important observational information for studies of jet kinematics otherwise supported by the bestobserved sample in the 15-GHz MOJAVE (Monitoring of Jets in Active Galactic Nuclei with VLBA Experiments) survey (Lister et al. 2009) restricted mainly to $z<2.5$. Reliable jet proper motion measurements could not yet be made at redshifts as high as $z \sim 5$. Information on the jet kinematics of J1430+4204 and other high-redshift sources would eventually be an important contribution for supplementing the apparent proper motionredshift statistics as a cosmological test (e.g. Cohen et al. 1988; Vermeulen \& Cohen 1994; Kellermann et al. 2004) with measurements at the extreme tail of the redshift distribution.

In this study, we report on our high-resolution radio interferometric observations of $\mathrm{J} 1430+4204$ (Sect. 2). We analyse the mas-scale radio structure of the source, and determine the Doppler boosting factor and the bulk Lorentz factor of the jet. We estimate the apparent proper motion of a possibly emerging new jet component and conclude that it is below the detection limit in our observations (Sect. 3). Finally, we put J1430+4204 in context by comparing it with other similar AGNs at lower redshifts (Sect. 4).

\section{Observations and data analysis}

We observed $\mathrm{J} 1430+4204$ at $15 \mathrm{GHz}$ with the ten $25-\mathrm{m}$ diameter radio telescopes of the US National Radio Astronomy Observatory (NRAO) Very Long Baseline Array (VLBA) on 2006 September 15 . The total observing time was $8 \mathrm{~h}$. The data were recorded in full circular polarisation with a bandwidth of $32 \mathrm{MHz}$. We also obtained data taken earlier in a similar $15-\mathrm{GHz}$ VLBA experiment (code BY019) from the NRAO data archive. These observations were done on 2005 February 23, prior to the peak of the total flux density curve (Fig. 1) of J1430+4204. The observing time was $5.8 \mathrm{~h}$. Both data sets were correlated at the NRAO VLBA correlator in Socorro (New Mexico, USA).
We performed the standard VLBI calibration procedures for both experiments using the NRAO Astronomical Image Processing System (AIPS, Diamond 1995). The visibility amplitudes were calibrated using system temperatures and antenna gains measured at the antennas. Instrumental delays were corrected using phase-calibration information provided along with the data. Fringe-fitting was performed over 2-min solution intervals. Additional calibrator and fringe-finder sources (J0854+2006, J0927+3902, J1256-0547, J1407+2827, $\mathrm{J} 1426+3625$ and $\mathrm{J} 1642+3948$ ) were also observed.

The calibrated visibility data were exported to and imaged with the Caltech Difmap package (Shepherd et al. 1994). The conventional hybrid mapping procedure with several iterations of CLEANing and phase (then amplitude) self-calibration was applied. Our uniformly-weighted total intensity images of J1430+4204 are displayed in Figs. 2a (2005 February 23) and 2b (2006 September 15). Uniform weighting was applied to maximise the angular resolution. Naturally-weighted images did not reveal any other low surface brightness structure. Identical coordinate scales and contour levels are used in both images. The fractional linear polarisation of the VLBI "core" was $\sim 1 \%$ and $2 \%$ at the first and second epochs, respectively. We detected no polarised emission from outside the core.

The source total flux density at $15 \mathrm{GHz}$ was monitored at the Ryle Telescope (Fabian et al. 1999). The light curve is shown in Fig. 1, where the epochs of the two VLBA experiments, as well as the flux densities measured with the VLBA are marked for comparison.

\section{Results}

\subsection{The compact radio structure of $\mathrm{J} 1430+4204$}

The mas-scale radio structure of $\mathrm{J} 1430+4204$ is dominated by a compact, nearly unresolved core in the 15-GHz VLBA images, at both epochs (Fig. 2). Additionally, a diffuse emission region 

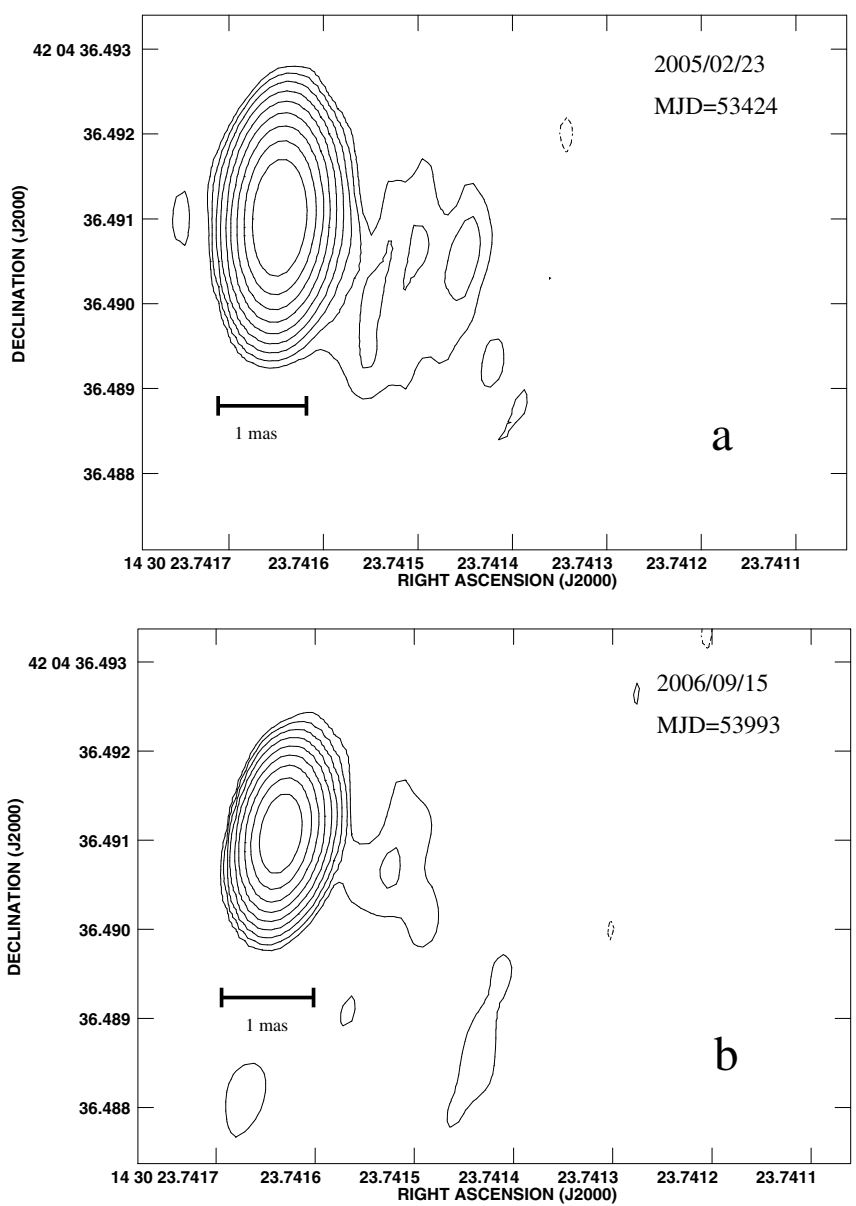

Fig. 2. 15-GHz VLBA images of J1430+4204 on 2005 February 23 a), top) and 2006 September 15 b), bottom). In the top image, the peak brightness is $200 \mathrm{mJy} / \mathrm{beam}$, the restoring beam is 1.17 mas $\times 0.52$ mas at the position angle $\mathrm{PA}=-3^{\circ} .8$. In the bottom image, the peak brightness is $159 \mathrm{mJy} / \mathrm{beam}$, the restoring beam is 1.22 mas $\times 0.58$ mas at $\mathrm{PA}=-11.95$. In both cases, the lowest contour levels are at $\pm 0.3 \mathrm{mJy} /$ beam, the positive contours increase by a factor of 2 .

is seen with a low surface brightness, out to $\sim 4$ mas from the brightness peak, in the position angles between about $-100^{\circ}$ and $-150^{\circ}$. (Position angles are conventionally measured from north through east.) This is most likely a steep-spectrum radio emission from optically thin plasma along the jet. But we do not have spectral information on the source at this spatial resolution, since our measurements were taken at the single frequency of $15 \mathrm{GHz}$. Notably, the position angle of the faint emission region is consistent with the source extension observed with VLBI at lower frequencies and lower resolution (USNO RRFID, Paragi et al. 1999; Helmboldt et al. 2007).

Apart from the compact core, we did not detect any significant jet component of which the proper motion could be reliably measured between the two observing epochs separated by 569 days.

We fitted elliptical Gaussian brightness distribution models to the radio core, using the self-calibrated visibility data in Difmap. The resulting model parameters are $S_{1}=209 \mathrm{mJy}$ flux density, $\vartheta_{1,1}=0.141$ mas major axis, $\vartheta_{2,1}=0.106$ mas minor axis (full width at half maximum, FWHM) at the first epoch, and $S_{2}=166 \mathrm{mJy}, \vartheta_{1,2}=0.143 \mathrm{mas}, \vartheta_{2,2}=0.059$ mas at the second epoch. The fitted major axis position angles $\left(-103^{\circ}\right.$ and $-101^{\circ}$ ) indicate a W-SW extension of the innermost radio structure, quite consistently with the weak mas-scale radio emission (Fig. 2).

The comparison of the total and VLBI flux densities at the first epoch (Fig. 1) indicates that $\sim 50 \mathrm{mJy}$ is completely resolved out by the VLBA. Although total flux density monitoring data were not available, by extrapolating the descending light curve, it is reasonable to assume a similar difference at the second VLBA epoch.

\subsection{Determination of the source parameters}

We used three methods for determining the following parameters of J1430+4204: the apparent transverse speed of a possible blob in the jet

$\beta_{\text {app }}=\frac{\beta \sin \theta}{1-\beta \cos \theta}$,

the Doppler boosting factor

$\delta=\frac{1}{\Gamma(1-\beta \cos \theta)}$,

and the bulk Lorentz factor

$\Gamma=\frac{\beta_{\text {app }}^{2}+\delta^{2}+1}{2 \delta}=\left(1-\beta^{2}\right)^{-\frac{1}{2}}$.

Here $\beta<1$ is the bulk speed of the material in the jet, expressed in the units of the speed of light $c$.

The most important physical parameter characterizing the jet flow is the bulk Lorentz factor $(\Gamma)$. We are also interested in the apparent tangential velocity of the putative blob in the jet which we translate into proper motion. The distance scale at this redshift is $6.497 \mathrm{pc}^{-1}$ mas $^{-1}$. We assume a flat cosmological model with $H_{0}=72 \mathrm{~km} \mathrm{~s}^{-1} \mathrm{Mpc}^{-1}, \Omega_{\Lambda}=0.73$ and $\Omega_{\mathrm{m}}=0.27$ throughout this paper.

Usually the Lorentz factor and the jet angle to the line of sight $(\theta)$ are determined from the measured Doppler factor, and the proper motion of jet component(s) inferred from multi-epoch VLBI monitoring of radio AGNs. Since the latter measurement is not available in our case, we took a different approach. For the jet angle to the line of sight, $\theta=\arctan \left(\frac{2 \beta_{\text {app }}}{\beta_{\text {app }}^{2}+\delta^{2}-1}\right)$, we assumed $3^{\circ}$ as found from bulk Comptonization modelling of the observed X-ray spectrum of J1430+4204 (Celotti et al. 2007). We determined the Doppler factor using three different methods, calculated the corresponding Lorentz factor, and estimated the amount of possible jet component proper motion. The inferred proper motion is indeed consistent with our non-detection. This in turn supports the initial assumption that $\theta$ has a small value and therefore this blazar is viewed nearly "pole-on".

\subsubsection{Parameters from the radio variability}

Hovatta et al. (2009) describe a method to determine the Doppler factor $\left(\delta_{\text {var }}\right)$ from the total flux density variability of the source. Following this method, we fitted an exponential function to the brightest flare in the flux density curve (Fig. 1) as

$\Delta S(t)=\left\{\begin{array}{l}\Delta S_{\max } \mathrm{e}^{\left(t-t_{\max }\right) / \tau}, \quad t<t_{\max } \\ \Delta S_{\max } \mathrm{e}^{\left(t-t_{\max }\right) / 1.3 \tau}, \quad t>t_{\max }\end{array}\right.$

where $t$ is the time, $t_{\max }$ is the epoch of the maximum flux density, $\Delta S_{\max }$ is the maximum amplitude (Jy), and $\tau$ is the rise time of the flare. For the major flare occurred in 2005, we obtained 


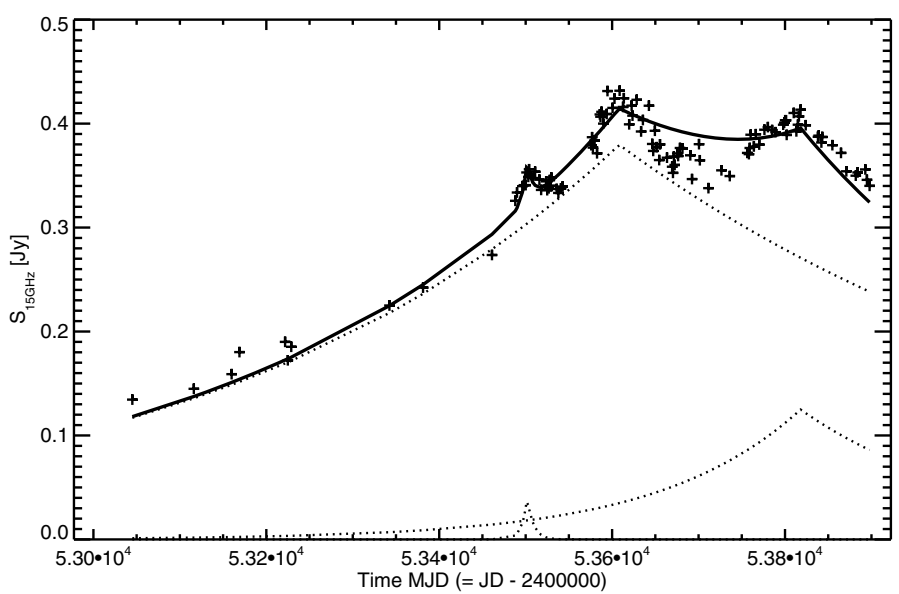

Fig. 3. The rising phase of the flare in the 15-GHz total flux density light curve (crosses) of J1430+4204, fitted with a three-component exponential model (solid curve). Dotted curves show the individual components.

$\Delta S_{\max }=0.380 \mathrm{Jy}$ and $\tau=478.4$ from fitting a three-component model to the light curve (Fig. 3).

We then determined the variability brightness temperature

$T_{\mathrm{b}, \mathrm{var}}=1.548 \times 10^{-32} \frac{\Delta S_{\max } d_{\mathrm{L}}^{2}}{v^{2} \tau^{2}(1+z)}$

where $d_{\mathrm{L}}^{2}$ is the luminosity distance of the source (m) and $v$ is the observing frequency $(\mathrm{GHz})$.

For calculating the Doppler factor from variability

$\delta_{\mathrm{var}}=\left(\frac{T_{\mathrm{b}, \mathrm{var}}}{T_{\mathrm{b}, \mathrm{int}}}\right)^{\frac{1}{3}}$,

we assume that energy equipartition holds between the particles and the magnetic field in the radio-emitting region (Readhead 1994). In this case the intrinsic brightness temperature is $T_{\mathrm{b}, \text { int }}=$ $5 \times 10^{10} \mathrm{~K}$.

We obtained the variability brightness temperature $T_{\mathrm{b}, \mathrm{var}}=$ $3.6 \times 10^{13} \mathrm{~K}$, and the Doppler factor $\delta_{\mathrm{var}}=9.0$. The implied apparent transverse speed is $\beta_{\text {app,var }}=2.2$ and thus the Lorentz factor is $\Gamma_{\mathrm{var}}=4.8$.

The apparent proper motion of a possible new jet component is $\mu=0.02$ mas $\mathrm{yr}^{-1}$. This means that during the time between the two VLBA observations $\left(\Delta t=569^{\mathrm{d}}\right)$ the blob could have moved by $\simeq 0.03$ mas. This angular displacement is just below the limit which we can possibly detect with the VLBA at this wavelength, about $10 \%$ of the restoring beam size (cf. Lister et al. 2009). Such a detection is further complicated by the fact that the absolute astrometric position of the core in our VLBA images is not known. Should the flare result in a slight displacement of the source brightness distribution peak (well within the restoring beam), the change remains unnoticed because self-calibration effectively shifts the brightness peak of the VLBA image into the phase centre, to the a priori source position that was assumed for the correlation of the interferometric measurements. Accurate relative astrometric registration of the brightness peak could have been possible via phase-referencing observations to an external radio source. Alternatively, a welldefined stationary feature further along the jet could have served as a positional reference, but there seems no suitable component in our images (Fig. 2).

\subsubsection{Parameters from the brightness temperature measured with VLBI}

The brightness temperature of a component in the radio source can be measured from our VLBI data, using the following formula (e.g. Condon et al. 1982):

$$
T_{\mathrm{b}, \mathrm{vlbi}}=1.22 \times 10^{12}(1+z) \frac{S}{\vartheta_{1} \vartheta_{2} v^{2}},
$$

where $S$ is the flux density (Jy), $\vartheta_{1}$ and $\vartheta_{2}$ are the major and minor axes of the fitted Gaussian model component (FWHM, mas). The $v$ observing frequency is expressed in $\mathrm{GHz}$.

We again assume that the intrinsic brightness temperature is the equipartition brightness temperature (Readhead 1994; Lähteenmäki et al. 1999), $T_{\mathrm{b} \text {,int }}=T_{\mathrm{eq}} \simeq 5 \times 10^{10} \mathrm{~K}$. We will call the Doppler boosting factor derived this way as the VLBI Doppler factor:

$\delta_{\mathrm{vlbi}}=\frac{T_{\mathrm{b}, \mathrm{vlbi}}}{T_{\mathrm{b}, \text { int }}}$.

This is valid if the brightness temperature is measured at the turnover frequency. In our case, the turnover frequency in the broad-band radio spectrum of is $\mathrm{J} 1430+4204$ is around $15 \mathrm{GHz}$ (Worsley et al. 2006), close to our VLBA observing frequency.

Our fitted elliptical Gaussian brightness distribution models to the core suggest $T_{\mathrm{b}, \text { lbi, } 1} \simeq 4.3 \times 10^{11} \mathrm{~K}$ and $T_{\mathrm{b}, \mathrm{vlbi}, 2} \simeq 6.0 \times$ $10^{11} \mathrm{~K}$ at the two VLBA epochs. These values correspond to Lorentz factors of $\Gamma_{1}=4.6$ and $\Gamma_{2}=6.8$ for the two epochs. Consistently with the first method based on variability, this one

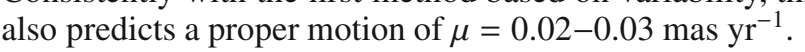

\subsubsection{Estimating the intrinsic brightness temperature}

So far we assumed that the intrinsic brightness temperature of the source was equal to the equipartition value, $T_{\text {eq }}=5 \times 10^{10} \mathrm{~K}$ (Readhead 1994). However, with two independent measurements of the apparent brightness temperature (from the variability and from the VLBI structure), and with the assumption that the underlying Doppler factors are the same, we can calculate the intrinsic brightness temperature (Lähteenmäki et al. 1999). Using Eqs. (6) and (8),

$T_{\mathrm{b}, \text { int }}=\left(T_{\mathrm{b}, \mathrm{vlbi}}\right)^{\frac{3}{2}}\left(T_{\mathrm{b}, \mathrm{var}}\right)^{-\frac{1}{2}}$.

At our two VLBI epochs, we get $T_{\mathrm{b}, \text { int, } 1}=4.7 \times 10^{10} \mathrm{~K}$ and $T_{\mathrm{b}, \text { int }, 2}=7.7 \times 10^{10} \mathrm{~K}$, respectively. Given the uncertainties in the estimates, these values are in very good agreement with the equipartition value.

\subsubsection{Parameters from the inverse Compton process}

By assuming that the X-ray radiation of the source results from inverse-Compton scattered photons of the radio synchrotron radiation, one can estimate the Doppler factor (Ghisellini et al. 1993; Güijosa \& Daly 1996) as

$\delta_{\mathrm{IC}}=f(\alpha)(1+z) S_{\mathrm{m}} \times\left[\frac{\ln \left(v_{\mathrm{b}} / v_{\mathrm{op}}\right) v_{\mathrm{x}}^{\alpha}}{S_{\mathrm{X}} \theta_{\mathrm{d}}^{6-4 \alpha} v_{\mathrm{op}}^{5-3 \alpha}}\right]^{1 /(4-2 \alpha)}$

where $f(\alpha)=-0.08 \alpha+0.14, S_{\mathrm{m}}$ is the radio flux density at the turnover frequency extrapolated from the optically thin part of the spectrum, $v_{\mathrm{b}}$ is the synchrotron high-frequency cutoff assumed to be $10^{5} \mathrm{GHz}$. The observed frequency of the radio peak is $v_{\mathrm{op}}=15.3 \mathrm{GHz}$. To apply this method, we also need X-ray 
Table 1. Parameters of $\mathrm{J} 1430+4204$ estimated from radio variability, VLBI imaging and the inverse Compton process.

\begin{tabular}{lcccc}
\hline \hline Method & $\delta$ & $\Gamma$ & $\beta_{\text {app }}$ & $\mu\left(\mathrm{mas} \mathrm{yr}^{-1}\right)$ \\
\hline var & 9.0 & 4.8 & 2.2 & 0.02 \\
VLBI & $8.6-12.0$ & $4.6-6.8$ & $2.0-4.2$ & $0.02-0.03$ \\
IC & $11.0-15.9$ & $6.1-10.3$ & $3.4-8.6$ & $0.03-0.07$ \\
\hline
\end{tabular}

data of the source which we took from Celotti et al. (2007). The X-ray flux density is $S_{\mathrm{X}}=2.32 \times 10^{-7} \mathrm{Jy}$ measured at $v_{\mathrm{X}}=1.25 \mathrm{keV}, \alpha=-0.38$ is the optically thin spectral index where the $S_{v} \propto v^{\alpha}$ convention is used, and $\theta_{\mathrm{d}}$ is the angular diameter of the source in mas. The fit for radio peak frequency and the extrapolation from the optically thin part was carried out using the data by Worsley et al. (2006).

Here we also need to assume that the VLBI observations were carried out at the turnover frequency, which holds for our case. We find the value of the inverse-Compton Doppler factor to be $\delta_{\mathrm{IC}, 1} \simeq 11$ and $\delta_{\mathrm{IC}, 2} \simeq 15.9$ for the first and second VLBA epochs, respectively. Assuming again $\theta=3^{\circ}$ (Celotti et al. 2007), the Lorentz factor and the apparent transverse speed for the two epochs are: $\Gamma_{\mathrm{IC}, 1} \simeq 6.1$ and $\Gamma_{\mathrm{IC}, 2} \simeq 10.3 ; \beta_{\mathrm{app}, 1}=3.4$ and $\beta_{\text {app }, 2}=8.6$. At the distance of the source, this trans-

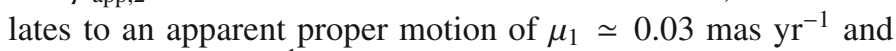

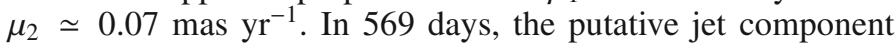
would have moved by $\simeq 0.04-0.1$ mas.

\section{Discussion}

We estimated the Doppler factor in the blazar J1430+4204 using different methods based on radio variability $\left(\delta_{\text {var }}\right)$, VLBI imaging assuming equipartition $\left(\delta_{\mathrm{vlbi}}\right)$, and the inverse Compton process $\left(\delta_{\text {IC }}\right)$. These estimates are summarised in Table 1 , along with the other source parameters derived.

While the mean $\delta_{\mathrm{vlbi}} / \delta_{\mathrm{IC}}$ ratio is in the order of unity for the AGN sample of Güijosa \& Daly (1996), there are differences up to a factor of $\sim 4$ in the Doppler boosting factors derived by the two independent methods. Errors come from the uncertainties of the input parameters, and larger inconsistencies may reflect deviations from the equipartition in certain sources. In the case of $\mathrm{J} 1430+4204$, our three estimates of the Doppler factor are remarkably similar, the values agree within a factor of $\sim 2$.

To estimate the bulk speed of the jet material $(\beta)$ and the corresponding Lorentz factor $(\Gamma)$, we assumed $\theta=3^{\circ}$ for the jet angle to the line of sight, found from bulk Comptonisation modelling of the X-ray spectrum by Celotti et al. (2007). At a given Doppler factor, $\sin \theta \leq 1 / \delta$ (e.g. Urry \& Padovani 1995), which in our case constrains the allowed range of the viewing angles to $\theta \lesssim 6^{\circ}$. The value of $\Gamma$ is quite insensitive to the actual $\theta$ in most of the possible range of viewing angles. Therefore our conclusions remain valid even if we allow for somewhat different values of $\theta$.

From measuring the source variability and from imaging the mas-scale radio structure at nearly the same time, we could determine the intrinsic brightness temperature of $\mathrm{J} 1430+4204$. Our values $\left(T_{\mathrm{b}, \text { int }, 1}=4.3 \times 10^{10} \mathrm{~K}\right.$ and $T_{\mathrm{b}, \text { int }, 2}=7.1 \times 10^{10} \mathrm{~K}$ at two different epochs) are consistent with those obtained for a larger sample of lower-redshift AGNs (Lähteenmäki et al. 1999), suggesting that $T_{\mathrm{b} \text {,int }} \lesssim 10^{11} \mathrm{~K}$ in compact extragalactic radio sources.

How is the other intrinsic parameter, the Lorentz factor of the jet in $\mathrm{J} 1430+4204(\Gamma \simeq 5-10)$ compared to those values measured in other AGNs? The systematic multi-epoch $15-\mathrm{GHz}$
VLBA monitoring of a large flux-density-limited sample of radio-loud AGNs in the 2-cm VLBA Survey and the MOJAVE survey (Kellermann et al. 2004; Cohen et al. 2007; Lister et al. 2009) revealed that Lorentz factors can be as high as $\Gamma \sim 50$, but the sources typically have values between 5 and 10 . The overwhelming majority of the MOJAVE sources are at $z<2.5$; the most distant object in the sample is J0646+4451 at $z=3.4$. As far as the Lorentz factor is concerned, our high-redshift object is therefore similar to those studied in the MOJAVE survey.

The maximum apparent speed vs. redshift plot for the MOJAVE quasars (Fig. 8 of Lister et al. 2009) shows that most of the values are found in the range $2<\beta_{\text {app }}<30$. The estimated apparent speed of $\mathrm{J} 1430+4204$ fits well in this picture and is closer to the low values.

The estimate for the jet apparent proper motion $(\mu \simeq$

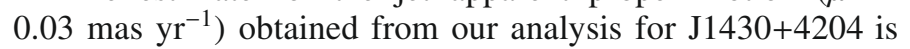
small compared to other sources at lower redshifts. This result fits the observed angular velocity-redshift relation and is consistent with the cosmological interpretation of the redshifts (Kellermann et al. 2004).

\section{Conclusion}

The high-redshift $(z=4.72)$ blazar $\mathrm{J} 1430+4204$ produced a major radio flux density outburst in 2005 (Fig. 1). About a year after the peak of the $15-\mathrm{GHz}$ flux density curve, we imaged the source with the VLBA at this frequency. We also analysed similar archive VLBA data taken during the rise of radio light curve. At both epochs separated by 569 days, the mas-scale radio structure of the source was remarkably similar: a compact optically thick "core" and a weak extension to W-SW (Fig. 2). The core could be fitted with elliptical Gaussian model components.

We searched for a new emerging component usually observable after large radio outbursts in AGNs. Based on our VLBA imaging, we did not detect any new jet component to be associated with the event. Assuming a small jet angle to the line of sight, we used three different methods to estimate the expected proper motion of such a component. These gave consistently small values for the proper motion. We found that our time base and angular resolution were insufficient to distinguish any new blob in the jet. Supposing that the jet was launched in 2004, the elapsed time until the second VLBA observation is 950 days. During this time the putative blob should have moved by 0.05 mas.

Our estimates for the intrinsic physical parameter describing the jet, the bulk Lorentz factor $(\Gamma \simeq 5-10)$ are comparable to the typical values found for other well-studied samples of blazars (e.g. Hovatta et al. 2009; Lister et al. 2009). The intrinsic brightness temperature is close to the equipartition value and is fully consistent with brightness temperatures measured in other sources $\left(T_{\mathrm{b}, \text { int }} \lesssim 10^{11} \mathrm{~K}\right.$, Lähteenmäki et al. 1999)

According to our estimates of the apparent proper motion, the emergence of a new jet component associated with the major 2005 outburst in J1430+4204 could possibly be detected with the angular resolution of ground-based VLBI at $15 \mathrm{GHz}$ over a time span of $\sim 10$ years.

Acknowledgements. The National Radio Astronomy Observatory (NRAO) is a facility of the National Science Foundation operated under cooperative agreement by Associated Universities, Inc. We are grateful to Guy Pooley for sharing the Ryle Telescope data with us. We thank the referee, Esko Valtaoja for his comments. This work was supported by the Hungarian Scientific Research Fund (OTKA grants K077795 and K72515). This research has made use of the United States Naval Observatory (USNO) Radio Reference Frame Image Database (RRFID), and the NASA/IPAC Extragalactic Database (NED) which 
is operated by the Jet Propulsion Laboratory, California Institute of Technology, under contract with the National Aeronautics and Space Administration.

\section{References}

Celotti, A., Ghisellini, G., \& Fabian, A. C. 2007, MNRAS, 375, 417 Cheung, C. C., Stawarz, L., Siemiginowska, A., et al. 2008, in Extragalactic Jets: Theory and Observation from Radio to Gamma Ray, ed. T. A. Rector, \& D. S. De Young, ASP Conf. Ser., 386, 462

Cohen, M. H., Barthel, P. D., Pearson, T. J., \& Zensus, J. A. 1988, ApJ, 329, 1

Cohen, M. H., Lister, M. L., Homan, D. C., et al. 2007, ApJ, 658, 232

Condon, J. J., Condon, M. A., Gisler, G., \& Puschell, J. J. 1982, ApJ, 252, 102

Diamond, P. J. 1995, in Very Long Baseline Interferometry and the VLBA, ed.

J. A. Zensus, P. J. Diamond, \& P. J. Napier, ASP Conf. Ser., 82, 227

Fabian, A. C., Celotti, A., Pooley, G., et al. 1999, MNRAS, 308, L6

Ghisellini, G., Padovani, P., Celotti, A., \& Maraschi, L. 1993, ApJ, 407, 65

Güijosa, A., \& Daly, R. A. 1996, ApJ, 461, 600
Helmboldt, J. F., Taylor, G. B., Tremblay, S., et al. 2007, ApJ, 658, 203 Hook, I. M., \& McMahon, R. G. 1998, MNRAS, 294, L7

Hovatta, T., Valtaoja, E., Tornikoski, M., \& Lähteenmäki, A. 2009, A\&A, 494, 527

Kellermann, K. I., Lister, M. L., Homan, D. C., et al. 2004, ApJ, 609, 539

Lähteenmäki, A., Valtaoja, E., \& Wiik, K. 1999, ApJ, 511, 112

Lister, M. L., Cohen, M. H., Homan, D. C., et al. 2009, AJ, 138, 1874

Paragi, Z., Frey, S., Gurvits, L. I., et al. 1999, A\&A, 344, 51

Readhead, A. C. S. 1994, ApJ, 426, 51

Savolainen, T., Wiik, K., Valtaoja, E., Jorstad, S. G., \& Marscher, A. P. 2002, A\&A, 394, 851

Shepherd, M. C., Pearson, T. J., \& Taylor, G. B. 1994, BAAS, 26, 987

Urry, C. M., \& Padovani, P. 1995, PASP, 107, 803

Vermeulen, R. C., \& Cohen, M. H. 1994, ApJ, 430, 467

Worsley, M. A., Fabian, A. C., Celotti, A., \& Iwasawa, K. 2004, MNRAS, 350, L67

Worsley, M. A., Fabian, A. C., Pooley, G. G., \& Chandler, C. J. 2006, MNRAS, 368,844 